\title{
BMJ Open Multimodal prehabilitation as strategy for reduction of postoperative complications after cardiac surgery: a randomised controlled trial protocol
}

Miquel Coca-Martinez, ${ }^{1}$ Antonio Lopez-Hernandez (D) , ${ }^{1}$ Mar Montane-Muntane, ${ }^{1}$ Maria Jose Arguis, ${ }^{1,2}$ Elena Gimeno-Santos, ${ }^{2,3}$ Ricard Navarro-Ripoll, ${ }^{1}$ Juan Perdomo, ${ }^{1}$ Manuel Lopez-Baamonde, ${ }^{1}$ Jose Rios, ${ }^{2,4}$ Jorge Moises, ${ }^{5}$ Maria Sanz de la Garza, ${ }^{6}$ Elena Sandoval, ${ }^{7}$ Barbara Romano, ${ }^{8}$ Raquel Sebio, ${ }^{3}$ Fernando Dana, ${ }^{3}$ Graciela Martinez-Palli ${ }^{1,2}$

To cite: Coca-Martinez M, Lopez-Hernandez A, MontaneMuntane M, et al. Multimodal prehabilitation as strategy for reduction of postoperative complications after cardiac surgery: a randomised controlled trial protocol. BMJ Open 2020;10:e039885. doi:10.1136/ bmjopen-2020-039885

- Prepublication history for this paper is available online. To view these files, please visit the journal online (http://dx.doi. org/10.1136/bmjopen-2020039885).

Received 04 May 2020

Revised 02 November 2020

Accepted 20 November 2020

Check for updates

(C) Author(s) (or their employer(s)) 2020. Re-use permitted under CC BY-NC. No commercial re-use. See rights and permissions. Published by BMJ.

For numbered affiliations see end of article.

Correspondence to Dr Graciela Martinez-Palli; GMARTIN@clinic.cat

\section{ABSTRACT}

Introduction Prehabilitation programmes that combine exercise training, nutritional support and emotional reinforcement (multimodal prehabilitation) have demonstrated efficacy reducing postoperative complications in the context of abdominal surgery. However, such programmes have seldom been studied in cardiac surgery, one of the surgeries associated with higher postoperative morbidity and mortality. This trial will assess the feasibility and efficacy in terms of reduction of postoperative complications and cost-effectiveness of a multimodal prehabilitation programme comparing to the standard of care in cardiac surgical patients.

Methods and analysis This is a single-centre, randomised, open-label, controlled trial with a 1:1 ratio. Consecutive 160 elective valve replacement and/ or coronary revascularisation surgical patients will be randomised to either standard of care or 4-6 weeks of multimodal prehabilitation that will consist in (1) two times/week supervised endurance and strength exercise training sessions, (2) promotion of physical activity and healthy lifestyle, (3) respiratory physiotherapy, (4) nutrition counselling and supplementation if needed, and (5) weekly mindfulness sessions. Baseline, preoperative and 3-month postoperative data will be collected by an independent blinded evaluator. The primary outcome of this study will be the incidence of postoperative complications.

Ethics and dissemination This study has been approved by the Ethics Committee of Clinical investigation of Hospital Clinic de Barcelona (HCB/2017/0708). The results will be disseminated in a peer-reviewed journal.

Trial registration number NCT03466606.

\section{INTRODUCTION}

\section{Background and rationale}

Patients undergoing cardiac surgery are the most likely to have complications (more than $20 \%$ ) and to die within the perioperative period (5.5\% perioperative mortality in Spain). ${ }^{1}$ The occurrence of postoperative complications and the risk of death are mainly conditioned by
Strengths and limitations of this study

- Scarce evidence of the benefits of multimodal prehabilitation in cardiac surgery population.

- First multimodal prehabilitation randomised controlled trial assessing postoperative complications as primary outcome in high-risk cardiac surgery patients.

- A decrease in postoperative complications could have a big impact in clinical practice, contributing to decrease length of stay and associated costs.

- Unmasked single-centre study. Nevertheless, assessments and postoperative complications will be collected by an independent blinded evaluator.

- Limited sample size of 160 patients to detect a reduction of postoperative complications of $20 \%$.

the magnitude of the surgical stress and by the patient's functional status, which is frequently conditioned by the presence of comorbidities. ${ }^{2}$ Despite the great advances that cardiac surgery and postoperative care have accomplished in the last decades, perioperative morbidity and mortality continue to be significant; mainly due to the increase of surgical indications in highrisk populations, older patients and patients with multiple comorbidities. The proportion of patients referred to cardiac surgery aged more than 75 years has risen from $16 \%$ in 1990 to $25 \%$, as shown in recent studies. ${ }^{3}$ Postsurgical complications have undeniable impact in quality of life, short-term and middle-term survival and economical cost. ${ }^{45}$ Many studies have demonstrated the association between frailty and morbidity, mortality and hospital length of stay. ${ }^{6}$ Frailty is defined as a state of major vulnerability to stressors which usually goes with sarcopenia, reduced strength, resistance and level of physical activity. ${ }^{7}$ It is estimated that $25 \%-50 \%$ of the 
candidates to cardiac surgery are frail. Frailty has been associated with a twofold to threefold increased risk of mortality and major morbidity after cardiac surgery and increased healthcare costs. ${ }^{78}$

Patients referred to cardiac surgery usually present a significant reduction in their functional capacity, which is multifactorial. The cardiovascular disease itself and its associated symptoms such as dyspnoea or angina pectoris, respiratory comorbidities, anaemia, malnourishment, muscle mass loss, depression or anxiety can be found among the factors causing a decline in functional capacity. ${ }^{9-12}$ All of the aforementioned factors induce a tendency to physical inactivity which further deteriorates functional capacity. This deconditioning places the patients at a higher risk of postoperative morbidity and mortality, complicating the recovery phase as well. A recent study in patients referred to oncological abdominal surgery showed that the preservation of physical activity was the only factor independently associated to lower postoperative morbidity and mortality, showing a higher predictive value than age and the presence of comorbidities. $^{13}$ This is concordant with numerous studies that relate physical activity and functional capacity with postoperative complications and postoperative recovery after major surgery. ${ }^{814} 15$ During the waiting period especially for surgery, physical inactivity is frequently more marked due to the fear that physical activity may exacerbate symptoms and also due to surgical anticipatory anxiety. Consequently, during this time span, there is a progressive deterioration in functional capacity which is not only reflected by a significant mortality rate among patients who are on the wait list but also by a prolongation of the patients' recovery. ${ }^{16}$ This prolonged postoperative period causes an increase in hospital length of stay, medication administration and delays patients' return to daily activities. Therefore, it has an impact on patients' quality of life and also on healthcare costs. ${ }^{45}$

The aim of preoperative identification of the highrisk candidates to cardiac surgery is the development of strategies to optimise their health status and functional capacity prior to surgery. ${ }^{17}$ Prehabilitation emerges as a promising preventive intervention ${ }^{18} 19$ carried out during the presurgical period, while the patient is on the waiting list. It integrates a programme of supervised physical exercise training, nutritional counselling and supplementation, and psychological support. ${ }^{2021}$ The goal of physical exercise training is to increase the patient's functional capacity in order to increase their physiological resistance to surgical stress. Nutritional counselling is an essential complement to physical exercise training in as much as it guides patients in losing weight if needed, promotes anabolism and it ensures adequate protein intake to counteract the catabolism induced by exercise training. ${ }^{22}$ Psychological support is a useful tool to reduce perioperative anxiety and depression, which in turn increases patient engagement to physical exercise. ${ }^{23}$ Then, prehabilitation would provide an approach, through risk reduction therapies and promotion of heart healthy behaviours, potentially beneficious preoperatively and postoperatively.

Although there is robust evidence regarding the effectiveness of prehabilitation in patients undergoing abdominal oncological surgery, ${ }^{1824-26}$ the role of prehabilitation programmes on reducing undesirable perioperative events has been assessed to a smaller extent in patients undergoing cardiac surgery. Arthur et $a l^{27}$ showed the efficacy of a multidimensional intervention in terms of intensive care unit (ICU) and hospital length of stay after coronary artery bypass grafting (CABG). However, this study was carried out in a group of low-risk patients and it did neither describe the preoperative intervention nor its impact on functional capacity. Even though the existing evidence hints at a beneficial effect of prehabilitation in cardiac surgery, the available literature is limited to low-risk patients and presents high heterogeneity on the intervention performed. ${ }^{28-30}$ This is a barrier to largescale implementation of prehabilitation programmes in candidates to cardiac surgery.

\section{Objectives}

The general aim of this study is to investigate whether multimodal prehabilitation is effective in reducing postoperative complications in patients undergoing elective coronary revascularisation and/or valve replacement surgery.

Secondary outcomes will include: severity of postoperative complications, impact of prehabilitation on functional capacity, anxiety and depression, physical activity, hospital length of stay, ICU stay and readmission rate and a cost-effectiveness analysis.

\section{Trial design}

The trial protocol was written following the Standard Protocol Items: Recommendations for Interventional Trials guidelines. ${ }^{31}$ This is a prospective, randomised, open-label, controlled trial with allocation ratio 1:1 comparing standard of care versus 4-6 weeks of a multimodal prehabilitation programme. The recruitment period is scheduled from March 2018 to January 2021. Both primary end-point and baseline data will be blindly assessed. Intention-to-treat criteria will be used in order to define the analysis population; all randomised patients will be included in the analysis according to assigned group. The study protocol and informed consent have been evaluated and accepted by the Medical Research Ethics Committee at Hospital Clinic de Barcelona (2017/0708). The trial will be conducted according to the rules of Good Clinical Practice.

\section{METHODS AND ANALYSIS \\ Setting}

This trial will be conducted in a single academic tertiary hospital in Barcelona, Spain (Hospital Clinic de Barcelona). Assessments and study interventions will be performed at the outpatient ambulatory infrastructure of Hospital Clinic 
de Barcelona. Surgery and postoperative hospitalisation will take place in the aforementioned hospital.

\section{Eligibility criteria}

Inclusion criteria

Patients older than 18 years old scheduled for CABG and/ or valve surgery, with an expected waiting time before surgery of 6 weeks or more and that accept to participate in this clinical trial.

\section{Exclusion criteria}

Functional or anatomical physical impairment that makes it impossible to complete the assessments and the prehabilitation programme, determined by an experienced physiotherapist in cardiopulmonary rehabilitation; cognitive impairment that would impede understanding of study procedures, informed consent or study questionnaires; cardiac instability; dynamic left ventricle outflow tract obstruction, proven exercise induced arrhythmias; other comorbidities that imply clinical instability; patient refusal to participate in the study or impossibility to attend supervised training sessions. Specifically, we will not exclude patients with severe pulmonary hypertension or severe valvular stenosis.

\section{Study outline}

Participant screening, recruitment, randomisation and informed consent (T-1)

Participant selection will begin after surgery is proposed by the cardiac surgeon and a referral for preoperative evaluation is done. Within 1 week of the referral patient will be seen by an anaesthesiologist who will screen for eligibility criteria and will explore the willing of participating in a study related with cardiopulmonary reserve and postoperative outcome. If eligibility criteria are met, patient will be approached by a research medical staff involved in the trial. The research medical staff in charge of obtaining informed consent will randomise prior to meeting with the patient and proposing study participation.

Participants will be randomised with $1: 1$ ratio by a computer-generated sequence using Macro-SCReN software V.4.8.1.8302 (2018 Elsevier).

\section{Baseline assessment (T0)}

After signing the informed consent all participants will undergo a full blinded baseline medical, functional, nutritional and psychological assessment. Allocation to study group will be revealed to the part of the research team that will carry out the intervention once baseline assessment is completed. The functional capacity assessment will be conducted by a physiotherapist on different days to avoid fatigue related to it, and will consist in a standard incremental cardiopulmonary exercise testing on cycloergometer (CPET), where physiological variables in response to exercise and workload in watts (W) will be measured at the anaerobic threshold and at $\mathrm{VO}_{2}$ peak. ${ }^{32-34}$ Endurance time $(\mathrm{ET})^{33}$ in seconds will be measured by a cycling constant work-rate exercise test performed at a load equivalent of $80 \%$ of the peak workload (PWR) the patient could tolerate on the incremental CPET (Ergoline 900, Ergoline, Bitz, Germany and Ergocard Professional, Medisoft, Sorinnes, Belgium). It will detect the responsiveness of interventions in terms of improved exercise capacity. Participants will also undergo hand grip strength test (Jamar Hydraulic Hand Dynamometer; Sammons Preston, Bolingbrook, Illinois, USA), $6 \mathrm{~min}$ walk test $(6 \mathrm{MWT})^{35}$ and sit-to-stand (STS) test. ${ }^{36}$ The $6 \mathrm{MWT}$ will assess functional capacity in relation to activities of daily living. Constant work rate exercise test and $6 \mathrm{MWT}$ are complementary in the comprehensive evaluation of these patients. Nutritional status will be assessed by a registered dietitian using the Patient Generated Subjective Global Assessment, weight, body mass index, prealbumin and glycosylated hemoglobin (HbAlc). American Society of Anesthesiologists status, Charlson Comorbidity Index ${ }^{37}$ and Euroscore $\mathrm{II}^{38}$ data will be recorded. Physical activity will be measured by the Yale Physical Activity Survey (YPAS) ${ }^{39}$ functional capacity by Dukes Activity Status Index questionnaire ${ }^{40}$ and Anxiety and depression measured using the Hospital and Anxiety Scale (HADS). ${ }^{41}$ All patients will be reassessed using the same tests before undergoing cardiac surgery (T7) and at 3 months postoperatively (T9).

\section{Procedures}

Standard of care (T1-T6)

Participants in the control group will follow the standard preoperative protocol at Hospital Clinic de Barcelona that includes physical activity recommendation, nutritional and smoking cessation advice. Moreover, patients suffering from iron-deficiency anaemia will receive intravenous iron infusion. ${ }^{42}$

\section{Multimodal Prehabilitation programme (PreHab) (T1-T6)}

Participants in the intervention group will undergo, in addition to the standard of care aforementioned, a 4-6week personalised multimodal prehabilitation programme. The interventions included will be patientcentred aiming to optimise patients' preoperative health status while enhancing their empowerment and engagement. The main components of the programme will consist in:

1. Supervised exercise training programme: 1-hour session, two sessions per week at the hospital outpatient gym facility conducted by a physiotherapist. Heart rate, blood pressure, oxygen saturation and perceived exertion rate using the modified Borg scale ${ }^{43}$ will be monitored throughout the training session.

i. Endurance training will be performed on stationary bicycle (Bike Forma; Technogym; Cesena, Italy) and will be tailored to the participants according to their PWR (baseline CPET). Each session includes 5 min of warm-up and 5 min of cool-down pedalling at $30 \%-40 \%$ of their PWR. An interval training consists in at least 5 bouts combining 2 min of moderate to high-intensity exercise (starting at $70 \%$ of PWR and progressing to 
90\%-100\% of PWR throughout the programme) interspersed with $3 \mathrm{~min}$ recovery periods at lower intensity $(40 \%-50 \%$ of the PWR). Progression during the sessions will be tailored on individual basis to maximise the training effect.

ii. Strength training will consist in 2-3 upper (pectorals and latissimus dorsi) and lower (quadriceps) limb exercises based on 2-3 sets of 8-12 repetitions avoiding valsalva's manoeuvres. The training is performed in a modular training station using external load (Plurima Multistation Wall, Technogym; Cesena, Italy). At the first session, the physiotherapist obtains for each muscle group, the tolerated weight for the eight-repetition maximum test, to establish the initial weight for the strength training. The weight and/or the number of repetitions will increase every week to ensure strength progression and maximise the training effect. The strength training is performed 2 days per week (in combination with the endurance training).

2. Promotion of physical activity and healthy lifestyle: a physiotherapist adequately trained in behavioural strategies will use motivational interviewing techniques to promote physical activity and healthy lifestyle. The motivational interview will focus on empathy, reflective listening and affirmation, and will address the patients' barriers and limitations related to physical activity, to get a change in behaviour.

3. Respiratory physiotherapy: patients will be instructed on daily breathing exercises (chest expansions, diaphragmatic respirations and deep inspirations) with a volumetric incentive spirometer (Coach 2; Smith Medical; London, UK), at least twice per day, 1-2 sets of 10-15 breathings for each exercise.

4. Nutrition counselling and supplementation: participants will meet with a registered dietitian that will assess their nutritional status and their diet based on a self-reported 3-day food diary. Nutritional teaching and a Mediterranean, well-balanced cardioprotective diet will be instructed. An adequate protein intake of $1.2-1.5 \mathrm{~g} / \mathrm{kg} /$ day will be aimed to meet the European Society for Clinical Nutrition and Metabolism recommendations. ${ }^{445}$ If patient does not reach the recommended daily protein intake with diet, protein supplementation with whey protein (Fresubin Protein powder, Fresenius Kabi España) will be provided to reach the aforementioned daily protein intake.

5. Weekly mindfulness sessions: Patients will be invited to attend 1 weekly hourly session of Mindfulness-Based Stress Reduction conducted by a registered psychologist. It will be strongly recommended to those who reported high rates of anxiety and depression on the baseline HADS.

Compliance to the prehabilitation programme will be calculated counting the number of supervised sessions attended out of the total intended sessions. The occurrence of any exercise-related adverse events during the prehabilitation period will be recorded and will be reported immediately to the principal investigator.

Preoperative (T7) and 3-month postoperative functional assessment (T9)

All participants' functional capacity will be reassessed before surgery and 3 months postoperative using ET, hand grip strength test, 6MWT and STS test. Anxiety and depression and physical activity questionnaires will be also recorded at these time points.

\section{Surgery and hospital admission (T8)}

Surgery will take place in the habitual waiting timeframe and will be performed by the regular cardiac surgery teams present in Hospital Clinic de Barcelona. Perioperative clinical protocols will be followed in a usual fashion independently of the group allocation or study participation. Hospital staff, anesthesiologists, surgeons, nurses and other people involved in patient care will be blinded to the study arm. Intraoperative complications or events as well as the need blood product transfusion or any deviation from normal course will be recorded in the intraoperative case report form (CRF).

Summary of flow chart is given in figure 1 .

The primary outcome of this study is the incidence of postoperative complications before patient discharge from the hospital. The type of complications included will be classified following the standards of the European Society of Anesthesiology and the European Society of Intensive Care medicine ${ }^{46}$ : Acute kidney injury, acute respiratory distress syndrome, arrhythmia, cardiac arrest, cardiogenic pulmonary oedema, deep vein thrombosis, delirium, gastrointestinal bleed, infection, source uncertain, laboratory confirmed bloodstream infection, myocardial infarction, pneumonia, paralytic ileus, postoperative haemorrhage, pulmonary embolism, stroke, surgical site infection and urinary tract infection.

The severity of complications will be also recorded, as a secondary outcome measure, following the ClavienDindo classification. ${ }^{47}$ To ensure accuracy of the primary endpoint, the data will be collected by a physician member of the team, every day according to standard clinical care, and then reviewed by another researcher. Both of them will be blinded to the study group to avoid bias. Patientchargeable costs (ie, pharmacy and blood bank); tariffchargeable costs (ie, medical care, diagnostic techniques, laboratories, interconsultations); other healthcare resources use (ie, ICU stay, total hospitalisation length of stay, readmissions, emergency room visits) and survival will be also assessed at 3 months and 1 year postcardiac surgery by chart review.

\section{Data collection management and monitoring}

All participant's de-identified data collected at different time points (table 1) will be entered by research staff members into Research Electronic Data Capture (REDCap) CRF via a secure webpage interface. Online data will be stored in a safe server located the Hospital 


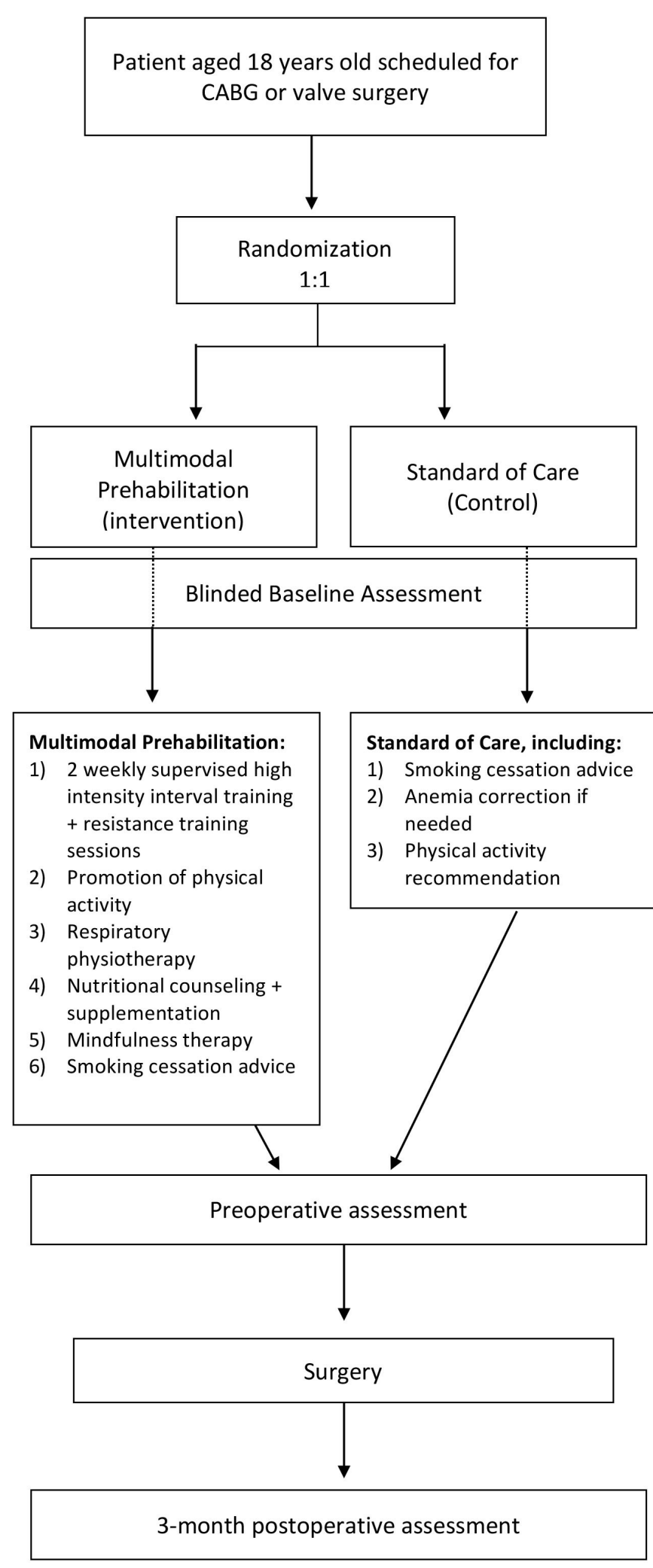

Figure 1 Flow chart. CABG, coronary artery bypass grafting.

Clinic facilities. All research team members will receive training on how to perform all the functional test included in the trial and how to input data in REDCap data manager.
Data monitoring

Previous experiences in prehabilitation studies show a clear absence of safety problems related to the experimental procedure. In addition, it is an unmasked trial, which is easier and faster to detect a problem and take appropriate measures.

The investigators of the present study are responsible for ensuring that the study meets the proposed milestones and deadlines. They will also be responsible for all aspects of the study design, management, ethical conduct, analysis and dissemination of results.

Safety data, including new hospitalisation, worsening angina or heart failure and arrhythmias, will be captured and all adverse events will be reported. They are responsible to periodically evaluate the study data for participant safety and study conduct, in addition to making modifications and/or termination of the trial after communication to the Ethics Committee of Clinical investigation of Hospital Clinic de Barcelona.

\section{Outcomes \\ Clinical outcomes}

The primary outcome variable of the study is the incidence of postoperative complications classified following the standards of the European Society of Anaesthesiology and European Society of intensive Care Medicine. ${ }^{46}$

Secondary outcome variables will be: (1) severity of postoperative complications using Dindo-Clavien classification; (2) hospital and ICU days of stay and (3) 3-month and 1-year mortality. Other outcome variables include: (1) ET measured by a cycling constant work-rate exercise testing at $80 \%$ of peak oxygen uptake; (2) distance covered in the 6MWT; (3) STS test and hand-grip strength; (4) physical activity by the YPAS and (5) hospitalisation direct costs and use of healthcare resources into the follow-up 1-year period after surgery.

Outcomes for feasibility of prehabilitation include incidence of adverse events during training, compliance to the supervised training sessions and adherence to the programme.

\section{Statistical analyses}

Sample size for this trial has been calculated based on the primary outcome of the study that is the reduction of incidence of postoperative complications in the prehabilitation group. Our own data in a similar population of patients indicated that the incidence of postoperative complications was $30 \%$. Accepting a two-sided alpha-risk of 0.05 and beta-risk of 0.20 , anticipating $10 \%$ of dropouts, indicated the need of including 80 participants per group to detect a reduction of the percentage of patients with complications in the intervention group compared with the control group $20 \%$. No adjustment for multiplicity was proposed due to this is a clinical trial with one primary outcome and without needed of interim analyses.

This trial will use intention-to-treat criteria for main analysis; nevertheless, analysis per protocol will also be performed as supportive analysis. Categorical variables, as 


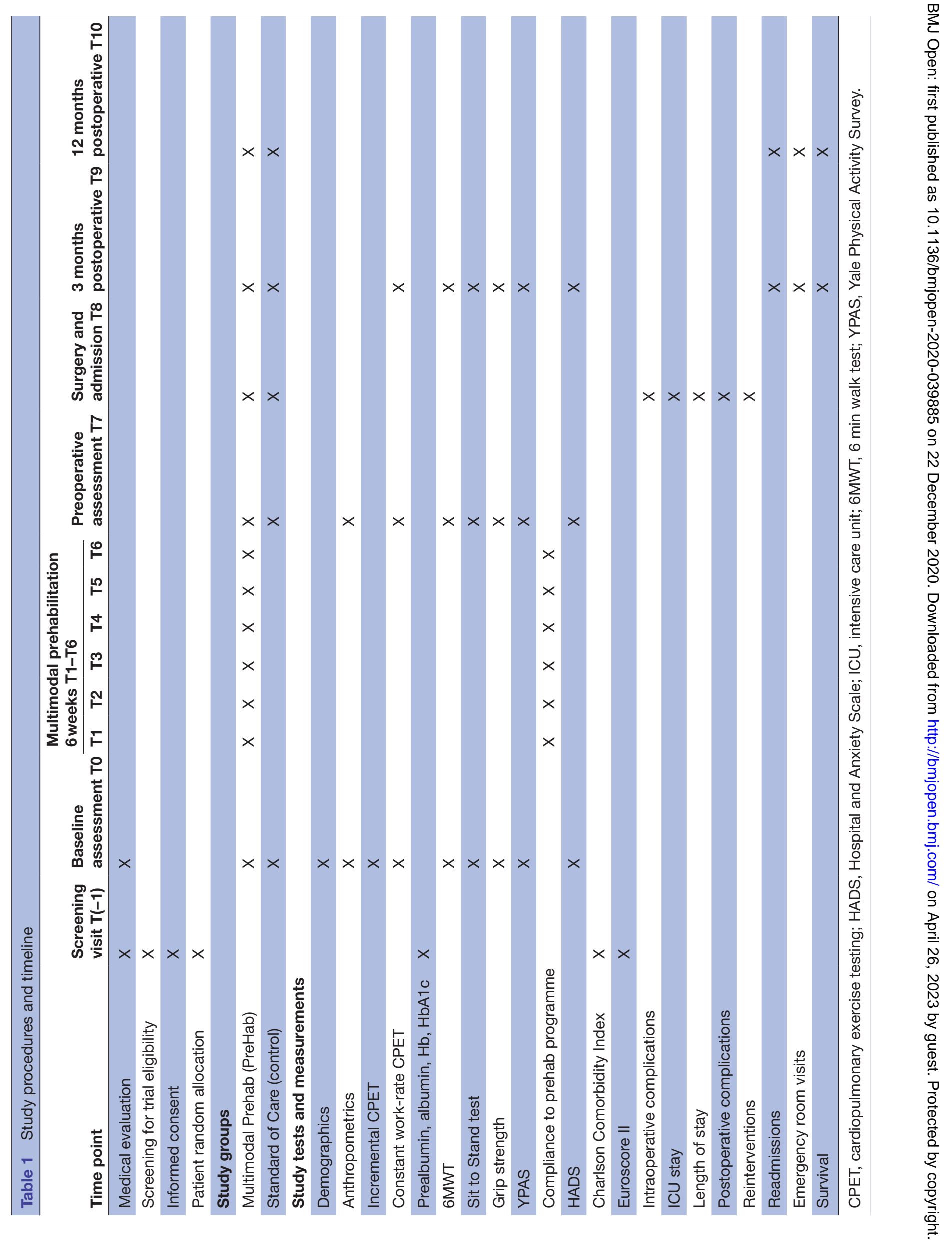


well primary outcome, will be analysed with Fisher's exact test. Continuous variables will be compared with Student's t-test for independent groups and Mann-Whitney $\mathrm{U}$ test according to each variable distribution. Ordinal variables will be analysed by Mann-Whitney $\mathrm{U}$ test.

Secondary outcomes looking at the difference between groups for the change in functional capacity over the different time points (repeated measures) will be analysed using generalised estimating equations models and will be shown as estimated effect and their 95\% CI. For these analyses, we will apply unstructured matrix in order to assess intra-subject correlation, for cases with no adjustments we will apply an autoregressive model (AR) (1) type matrix.

A cost-effectiveness study will be carried out from the perspective of the hospital, taking into account the costs of the intervention and the expenses related to the disease during the follow-up (30 days). For costs, the mean or median and their $95 \% \mathrm{CI}$ of difference in per-patient costs between the two groups will be computed (control-group costs minus prehabilitation-group costs), so that positive values will be interpreted as savings of the prehabilitation programme. Considering previous experience with prehabilitation cost analysis, ${ }^{19}$ a highly skewed distribution will probably be present. Right-sided asymmetric distribution appears when some patients incur in high costs, mainly because of major medical complications. To deal with this, a non-parametric approach (bootstrapping) will be used. Bootstrap analysis yields more robust when dealing with skewed cost data compared with non-parametric tests.

\section{Patient and public involvement}

Patient and public involvement was taken into consideration for the development of this protocol. A satisfaction questionnaire was given to a sample of patients who had undergone a prehabilitation programme as part of their clinical pathway before an abdominal surgery. They gave feedback about both the assessments and interventions included in this trial. As a result of their feedback we proceeded to reduce the number of questionnaires given during the assessments, as well as an assessment and intervention redesign, resulting in a more flexible schedule for the participants.

\section{ETHICS AND DISSEMINATION}

Ethics approval for this trial has been obtained by the Ethics Committee of Clinical investigation of Hospital Clinic de Barcelona (HCB $/ 2017 / 0708)$. This trial will be conducted according to the principles emanating from the Helsinki Declaration, with compliance to the Good Clinical Practice and the International Council for Harmonisation of Technical Requirements for Pharmaceuticals for Human Use (ICH) guidelines. Confidentiality for participants and their data will be guaranteed according to the Spanish data protection law 'Ley Orgánica de Protección de Datos de Carácter Personal' (15/1999, 13 diciembre) and the General Data Protection Regulation from the European Union (EU 2016/679).
All the explorations and tests in this trial are considered minimally invasive and are performed in presence of a physician under strict monitoring. The non-pharmacological characteristics of the intervention exclude the need for additional trial insurances.

\section{Informed consent}

To avoid contamination among groups, there will be two informed consents: all participants allocated in the control group will be proposed to participate in a study to investigate the relationship between cardiopulmonary reserve and postoperative outcomes and will sign the informed consent for the control group. All participants allocated in the intervention group will be proposed to participate in a study that investigates the effects of a multimodal prehabilitation programme on postoperative outcomes. Patients in the intervention group will sign the intervention group informed consent, where the intervention is defined. All potential participants will be given time to read the informed consent form, consider participation and ask doubts or questions regarding the study participation. If patient agrees to participate, he or she will be asked to provide written consent in the informed consent document. Participants will be free to withdraw their consent and opt out from the trial at any time without needing to explain the reason why. Withdrawal from the trial will not carry any prejudice in the participant care.

\section{Author affiliations \\ ${ }^{1}$ Anaesthesiology and Intensive Care, Hospital Clinic de Barcelona, Barcelona, Catalunya, Spain \\ ${ }^{2}$ August Pi i Sunyer Biomedical Research Institute (IDIBAPS), Barcelona, Catalunya, Spain \\ ${ }^{3}$ Prehabilitation Unit, Hospital Clinic de Barcelona, Barcelona, Catalunya, Spain ${ }^{4}$ Biostatistics Unit, Universitat Autonoma de Barcelona Facultat de Medicina, Bellaterra, Catalunya, Spain \\ ${ }^{5}$ Respiratory Medicine, Hospital Clinic de Barcelona, Barcelona, Catalunya, Spain \\ ${ }^{6}$ Department of Cardiology, Cardiovascular Institute, Hospital Clinic de Barcelona, Barcelona, Catalunya, Spain \\ ${ }^{7}$ Department of Cardiac Surgery, Cardiovascular Institute, Hospital Clinic de Barcelona, Barcelona, Catalunya, Spain \\ ${ }^{8}$ Nutrition and Clinical Dietetics, Hospital Clinic de Barcelona, Barcelona, Catalunya, Spain}

Contributors MC-M, EG-S, AL-H, MSdIG, RN-R, MJA and GM-P contributed to trial design and conception. MJA, GM-P colead investigators, obtained ethics approval. MC-M, MM-M, AL-H, MJA, JR and GM-P contributed to manuscript conception and drafting. EG-S, MSdIG, ES, BR, RN-R, FD, JM, RS, JP contributed to manuscript revision and edition. MC-M, MM-M, AL-H, EG-S, MSdIG, ES, BR, RN-R, FD, JM, RS, $J P, M L-B, M J A$ and GM-P approved the final version of the manuscript.

Funding Funding for the trial was provided by a grant from the Spanish Public Government Fondos de Investigación en Salud (FIS) Instituto Carlos III (PI17/00852), after a peer-reviewed process. The grant was awarded as a mean for the implementation of multimodal prehabilitation in cardiac surgery and the present trial is the main receiver of its funds along with a subproject of prehabilitation for cardiac transplant candidates. Protein supplementation with Fresubin Protein powder was provided and supported by Fresenius Kabi España.

Disclaimer The funding sources had no role in the design of this study and will not have any role during its execution, analyses or interpretation of the data.

Competing interests All researchers and authors of this protocol declare that they have no conflict of interest.

Patient and public involvement Patients and/or the public were not involved in the design, or conduct, or reporting, or dissemination plans of this research. 
Patient consent for publication Not required.

Provenance and peer review Not commissioned; externally peer reviewed.

Open access This is an open access article distributed in accordance with the Creative Commons Attribution Non Commercial (CC BY-NC 4.0) license, which permits others to distribute, remix, adapt, build upon this work non-commercially, and license their derivative works on different terms, provided the original work is properly cited, appropriate credit is given, any changes made indicated, and the use is non-commercial. See: http://creativecommons.org/licenses/by-nc/4.0/.

\section{ORCID iD}

Antonio Lopez-Hernandez http://orcid.org/0000-0002-5310-030X

\section{REFERENCES}

1 Polo López L, Centella Hernández T, López Menéndez J, et al. Cirugía cardiovascular en España en El año 2015. Registro de intervenciones de la Sociedad Española de Cirugía TorácicaCardiovascular. Cir cardiov 2016;23:289-305.

2 Moonesinghe SR, Mythen MG, Grocott MPW. High-risk surgery: epidemiology and outcomes. Anesth Analg 2011;112:891-901.

3 Afilalo J, Mottillo S, Eisenberg MJ, et al. Addition of frailty and disability to cardiac surgery risk scores identifies elderly patients at high risk of mortality or major morbidity. Circ Cardiovasc Qual Outcomes 2012;5:222-8.

4 Vonlanthen R, Slankamenac K, Breitenstein S, et al. The impact of complications on costs of major surgical procedures: a cost analysis of 1200 patients. Ann Surg 2011;254:907-13.

5 Lee L, Tran T, Mayo NE, et al. What does it really mean to "recover" from an operation? Surgery 2014;155:211-6.

6 Lee DH, Buth KJ, Martin B-J, et al. Frail patients are at increased risk for mortality and prolonged institutional care after cardiac surgery. Circulation 2010;121:973-8.

7 Fried LP, Ferrucci L, Darer J, et al. Untangling the concepts of disability, frailty, and comorbidity: implications for improved targeting and care. J Gerontol A Biol Sci Med Sci 2004;59:M255-63.

8 Afilalo J, Eisenberg MJ, Morin J-F, et al. Gait speed as an incremental predictor of mortality and major morbidity in elderly patients undergoing cardiac surgery. J Am Coll Cardiol 2010;56:1668-76.

9 Foster C, Haviland J, Winter J, et al. Pre-Surgery depression and confidence to manage problems predict recovery trajectories of health and wellbeing in the first two years following colorectal cancer: results from the crew cohort study. PLoS One 2016;11:e0155434.

10 Wilson RJT, Davies S, Yates D, et al. Impaired functional capacity is associated with all-cause mortality after major elective intraabdominal surgery. Br J Anaesth 2010;105:297-303.

11 Wolfe RR. The underappreciated role of muscle in health and disease. Am J Clin Nutr 2006;84:475-82.

12 Chermesh I, Hajos J, Mashiach T, et al. Malnutrition in cardiac surgery: food for thought. Eur J Prev Cardiol 2014;21:475-83.

13 Dronkers JJ, Chorus AMJ, van Meeteren NLU, et al. The association of pre-operative physical fitness and physical activity with outcome after scheduled major abdominal surgery. Anaesthesia 2013;68:67-73.

14 Snowden CP, Prentis J, Jacques B, et al. Cardiorespiratory fitness predicts mortality and hospital length of stay after major elective surgery in older people. Ann Surg 2013;257:999-1004.

15 Shulman MA, Cuthbertson BH, Wijeysundera DN, et al. Using the 6-minute walk test to predict disability-free survival after major surgery. Br J Anaesth 2019;122:111-9.

16 Sobolev BG, Levy AR, Kuramoto L, et al. The risk of death associated with delayed coronary artery bypass surgery. BMC Health Serv Res 2006;6:85.

17 Carli F, Minnella EM. Preoperative functional assessment and optimization in surgical patient: changing the paradigm. Minerva Anestesiol 2017;83:214-8.

18 Barberan-Garcia A, Ubré M, Roca J, et al. Personalised prehabilitation in high-risk patients undergoing elective major abdominal surgery: a randomized blinded controlled trial. Ann Surg 2018;267:50-6.

19 Barberan-Garcia A, Ubre M, Pascual-Argente N, et al. Post-discharge impact and cost-consequence analysis of prehabilitation in high-risk patients undergoing major abdominal surgery: secondary results from a randomised controlled trial. Br J Anaesth 2019;123:450-6.

20 Bousquet-Dion G, Awasthi R, Loiselle Sarah-Ėve, et al. Evaluation of supervised multimodal prehabilitation programme in cancer patients undergoing colorectal resection: a randomized control trial. Acta Oncol 2018;57:849-59.
21 van Rooijen SJ, Molenaar CJL, Schep G, et al. Making patients fit for surgery: introducing a four Pillar multimodal prehabilitation program in colorectal cancer. Am J Phys Med Rehabil 2019;98:888-96.

22 Gillis C, Carli F. Promoting perioperative metabolic and nutritional care. Anesthesiology 2015;123:1455-72.

23 Miró J, Raich RM. Preoperative preparation for surgery: an analysis of the effects of relaxation and information provision. Clin Psychol Psychother 1999;6:202-9.

24 Trépanier M, Minnella EM, Paradis T, et al. Improved disease-free survival after prehabilitation for colorectal cancer surgery. Ann Surg 2019;270:493-501.

25 Minnella EM, Awasthi R, Loiselle S-E, et al. Effect of exercise and nutrition prehabilitation on functional capacity in esophagogastric cancer surgery: a randomized clinical trial. JAMA Surg 2018;153:1081-9.

26 Minnella EM, Bousquet-Dion G, Awasthi R, et al. Multimodal prehabilitation improves functional capacity before and after colorectal surgery for cancer: a five-year research experience. Acta Oncol 2017;56:295-300.

27 Arthur HM, Daniels C, McKelvie R, et al. Effect of a preoperative intervention on preoperative and postoperative outcomes in low-risk patients awaiting elective coronary artery bypass graft surgery. A randomized, controlled trial. Ann Intern Med 2000;133:253-62.

28 Hulzebos EHJ, Helders PJM, Favié NJ, et al. Preoperative intensive inspiratory muscle training to prevent postoperative pulmonary complications in high-risk patients undergoing CABG surgery: a randomized clinical trial. JAMA 2006;296:1851-7.

29 Waite I, Deshpande R, Baghai M, et al. Home-based preoperative rehabilitation (prehab) to improve physical function and reduce hospital length of stay for frail patients undergoing coronary artery bypass graft and valve surgery. J Cardiothorac Surg 2017;12:91.

30 Sawatzky J-AV, Kehler DS, Ready AE, et al. Prehabilitation program for elective coronary artery bypass graft surgery patients: a pilot randomized controlled study. Clin Rehabil 2014;28:648-57.

31 Chan A-W, Tetzlaff JM, Altman DG, et al. SPIRIT 2013 statement: defining standard protocol items for clinical trials. Ann Intern Med 2013;158:200-7.

32 West MA, Asher R, Browning M, et al. Validation of preoperative cardiopulmonary exercise testing-derived variables to predict in-hospital morbidity after major colorectal surgery. Br J Surg 2016;103:744-52.

33 American Thoracic Society, American College of Chest Physicians. ATS/ACCP statement on cardiopulmonary exercise testing. Am J Respir Crit Care Med 2003;167:211-77.

34 Levett DZH, Jack S, Swart M, et al. Perioperative cardiopulmonary exercise testing (CPET): consensus clinical guidelines on indications, organization, conduct, and physiological interpretation. $\mathrm{Br} J$ Anaesth 2018;120:484-500.

35 ATS Committee on Proficiency Standards for Clinical Pulmonary Function Laboratories. ATS statement: guidelines for the six-minute walk test. Am J Respir Crit Care Med 2002;166:111-7.

36 Jones CJ, Rikli RE, Beam WC. A 30-S chair-stand test as a measure of lower body strength in community-residing older adults. Res $Q$ Exerc Sport 1999;70:113-9.

37 Charlson ME, Pompei P, Ales KL, et al. A new method of classifying prognostic comorbidity in longitudinal studies: development and validation. J Chronic Dis 1987;40:373-83.

38 Nashef SAM, Roques F, Sharples LD, et al. EuroSCORE II. Eur J Cardiothorac Surg 2012;41:734-45.

39 Donaire-Gonzalez D, Gimeno-Santos E, Serra I, et al. Validation of the Yale Physical Activity Survey in chronic obstructive pulmonary disease patients]. Arch Bronconeumol 2011;47:552-60.

40 Struthers R, Erasmus P, Holmes K, et al. Assessing fitness for surgery: a comparison of questionnaire, incremental shuttle walk, and cardiopulmonary exercise testing in general surgical patients. $\mathrm{Br}$ $J$ Anaesth 2008;101:774-80.

41 Snaith RP. The hospital anxiety and depression scale. Health Qual Life Outcomes 2003;1:29.

42 Munting KE, Klein AA. Optimisation of pre-operative anaemia in patients before elective major surgery - why, who, when and how? Anaesthesia 2019;74 Suppl 1:49-57.

43 Borg G. Psychophysical scaling with applications in physical work and the perception of exertion. Scand J Work Environ Health 1990;16 Suppl 1:55-8.

44 Weimann A, Braga M, Carli F, et al. ESPEN guideline: clinical nutrition in surgery. Clin Nutr 2017;36:623-50.

45 Gillis C, Loiselle S-E, Fiore JF, et al. Prehabilitation with whey protein supplementation on perioperative functional exercise capacity in patients undergoing colorectal resection for cancer: a pilot doubleblinded randomized placebo-controlled trial. J Acad Nutr Diet 2016;116:802-12. 
46 Jammer I, Wickboldt N, Sander M, et al. Standards for definitions and use of outcome measures for clinical effectiveness research in perioperative medicine: European perioperative clinical outcome (EPCO) definitions: a statement from the ESA-ESICM joint
Taskforce on perioperative outcome measures. Eur $\mathrm{J}$ Anaesthesiol 2015;32:88-105.

47 Dindo D, Demartines N, Clavien P-A. Classification of surgical complications: a new proposal with evaluation in a cohort of 6336 patients and results of a survey. Ann Surg 2004;240:205-13. 